\title{
Distale tiefe Venenthrombosen zeigen ähnliche Outcomes wie proximal gelegene
}

Vlazny D et al. Outcome of anticoagulation in isolated distal deep vein thrombosis compared to proximal deep venous thrombosis. J Thromb Haemost 2021; doi:10.1111/jth.15416

Tiefe Venenthrombosen (TVT) der unteren Extremität werden gewöhnlich in distale (nur Venen des Unterschenkels betroffen) und proximale TVT (Ausdehnung bis in den Oberschenkel bzw. nur Oberschenkelvenen betroffen) eingeteilt. Dabei gelten die distalen TVT oftmals als weniger gefährlich, was Rezidive, schwere Blutungen unter Therapie und Todesfälle betrifft.

Daher empfehlen Leitlinien bei dieser distalen TVT-Lage nicht unbedingt eine primäre Antikoagulation, sondern lassen auch (zunächst) eine regelmäßige sonografische Kontrolle zu. Nur: Für dieses "gutartigere" Verhalten distaler TVT gibt es keine harten Belege. Mediziner der Mayo Clinic haben sich daher 2 Registerdatenbanken näher angesehen: die des Mayo Clinic Gonda Vascular Center (März 2015 bis August 2018) und die des VTE Registry (März 2013 bis August 2020).

Dabei identifizierten Danielle Vlazny und ihre Kollegen fast 2000 Patienten, bei denen in diesem Zeitraum eine akute TVT mittels Sonografie gesichert worden war. Darunter waren

- 746 Patienten mit isolierter distaler TVT (Durchschnittsalter 62,4 Jahre;

46,1\% Frauen; Gruppe 1) und

- 1176 Patienten mit proximaler TVT

(Durchschnittsalter 62,8 Jahre, 39,8\%

Frauen; Gruppe 2).

Dabei waren auslösende Faktoren, etwa kurz zurückliegende Operationen, Traumata oder Bettlägerigkeit, in Gruppe 1 häufiger, während spontane Thrombosen und anamnestisch bekannte venöse Thromboembolien in Gruppe 2 häufiger zu verzeichnen waren, ebenso aktive Malignomerkrankungen.

Als Endpunkte beurteilten die Wissenschaftler Todesfälle und Rezidive, hier defi- niert als Ausdehnung des Thrombus nach proximal, neu auftretende TVT und neu auftretende Lungenembolien, und verglichen die Raten zwischen den beiden Gruppen. Primäre Sicherheitsendpunkte umfassten schwere Blutungen und klinisch relevante, nicht schwere Blutungen nach den Definitionen der International Society of Thrombosis Haemostasis.

Alle Patienten erhielten eine Antikoagulation mit entweder Heparin bzw. Warfarin oder mit direkten oralen Antikoagulanzien (Rivaroxaban, Apixaban). Dabei wurde unfraktioniertes Heparin etwas häufiger in Gruppe 1 eingesetzt (8,3\% vs. 2,0\%), Warfarin dagegen häufiger in Gruppe 2 (13,8\% vs. $10,6 \%$ ). Alle anderen Antikoagulanzien waren zwischen den beiden Gruppen ähnlich verteilt. Insgesamt machten fraktioniertes Heparin und die beiden direkten oralen Antikoagulanzien die große Mehrzahl der Behandlungen aus $(>80 \%$ in beiden Gruppen). Patienten mit proximalen TVT wurden signifikant länger antikoaguliert als Patienten mit distalen TVT (190,4 vs. 152,5 Tage).

Bei der Auswertung zeigte sich eine vergleichbare Rezidivrate zwischen den beiden Gruppen, mit

- 4,6 pro 100 Personenjahre in Gruppe 1,

- davon beinhalteten $60 \%$ eine Lungenembolie, und

- 5,77 pro 100 Personenjahre in Gruppe 2, - mit Lungenembolien in 39,5\% der Fälle.

Die Dauer bis zum Auftreten des Rezidivs variierte stark (zwischen 115 und 191 Tage).

Auffälligerweise starben in Gruppe 1 bis Monat 3 mehr Patienten als in Gruppe 2 ( $7,2 \%$ vs. $3,9 \%$ ), dieser Unterschied glich sich aber in der Folgezeit aus. Nach 6 Monaten und insgesamt zeigten sich keine signifikanten Unterschiede mehr (Gesamttodes- fallrate 31,89 pro 100 Personenjahre vs. 28,36 pro 100 Personenjahre in Gruppe 1 bzw. Gruppe 2). In der multivariaten Analyse errechneten die Forscher als unabhängige Prädiktoren für die Sterblichkeit:

- die Verwendung von Heparin bzw.

Warfarin (vs. direkte orale Antikoagulanzien),

- zunehmendes Alter und

- aktives Malignom.

Bei Patienten mit proximaler TVT erwies sich darüber hinaus ein höherer BodyMass-Index als protektiv.

Schwere bzw. klinisch relevante Blutungen traten in beiden Gruppen vergleichbar häufig auf.

Bei Subgruppenanalysen stellten die Mediziner fest, dass bei Verwendung von direkten oralen Antikoagulanzien (440 Patienten in Gruppe 1, 755 Patienten in Gruppe 2) die Rezidivrate in Gruppe 1 geringer war $(0,9 \%$ vs. $3,0 \%)$. Bei den anderen Substanzen fand sich kein Unterschied. Und auch wenn man innerhalb der beiden Gruppen die Rezidive betrachtete, erwiesen sich Rivaroxaban bzw. Apixaban als überlegen. Ähnliches galt für die Outcomes Blutungen und Tod.

FAZIT

Distale TVT sind insgesamt nicht weniger riskant als proximale TVT, was das Auftreten von Rezidiven betrifft, fassen die Autoren zusammen. Bei beiden Entitäten schnitt die Behandlung mit direkten oralen Antikoagulanzien besser ab als die mit Heparin oder Warfarin.

Dr. Elke Ruchalla, Bad Dürrheim 